\title{
Arbor
}

\section{Madrid, capital económica}

\author{
José Luis García Delgado
}

Arbor CLXIX, 666 (Junio 2001), 359-369 pp.

Con menos de un 2 por 100 del territorio nacional, pero dando cobijo al 13 por 100 de la población y generando el 16 por 100 del producto interior bruto total, Madrid, en el centro de la superficie peninsular ibérica y con un nivel de renta por habitante igual a la media de la Unión Europea, es, al cerrarse el siglo XX, no sólo la indiscutida capital económica de España, sino también una pieza estratégica y un componente de primer orden de la total estructura económica española: el resultado de un proceso cuyo arranque definitivo puede fecharse precisamente hace unos cien años, en el tiempo intersecular a caballo del ochocientos y del novecientos.

Resulta por eso incitante volver sobre los factores y los hechos que explican ese lugar prominente que va a alcanzar Madrid en los primeros compases de la centuria ahora clausurada. Una mirada hacia atrás - como la que quieren facilitar estas páginas- que tiene la ventaja, a la hora de interpretar el curso de la historia, de conocer la realidad en que éste desemboca. No estará de más, por ello, como prólogo de lo que después se dice, subrayar ahora los rasgos y funciones que acaban definiendo la condición de Madrid como capital económica de España conforme ha avanzado el siglo XX.

En el marco del crecimiento económico moderno en España, y particularmente a lo largo del siglo XX, Madrid, en efecto, no ha dejado de subir peldaños hasta colocarse a la cabeza del conjunto nacional, y no sólo atendiendo a los niveles comparados de bienestar material, sino también a las oportunidades de progreso y prosperidad que, hasta donde es razonable prever, el futuro puede brindar. Dicho de otro modo: ha sido la economía de Madrid la que, en el conjunto español, acaso mejor ha sabido adaptarse a las cambiantes circunstancias que han 
prevalecido a lo largo de la centuria del novecientos, poniendo de manifiesto una alta capacidad de cambio, de metamorfosis, incluso, aprovechando las condiciones nuevas creadas en cada momento para desempeñar funciones complementarias y diferentes en los engranajes de toda la economía de España. De suerte que su trayectoria, la trayectoria que describe la economía de Madrid a lo largo del último siglo, aunque no rectilínea y sin discontinuidades, acaba ofreciendo, probablemente más que ninguna otra en el mapa español, un perfil ascendente, afirmado con fuerza tanto en los decenios de 1950 y 1960 como en los dos que cierran el siglo, en el marco de la democracia y de las recuperadas libertades; trayectoria que está demostrando renovado brío, especial intensidad de nuevo durante el lustro con el que el novecientos ha dado la última vuelta de su camino.

Hay que subrayar esa capacidad adaptativa y de cambio del Madrid contemporáneo, porque en ella reside tal vez uno de sus rasgos identificadores. En la España decimonónica, la que contempla el rezagado arranque y luego el desigual desarrollo de la primera revolución industrial, Madrid, comenzará ya, aunque con tono moderado, a dar señales de cambio y remoción de situaciones heredadas. Y así, en los últimos compases del siglo XIX, superadas viejas barreras para la ampliación del suelo urbano, para el aprovisionamiento de agua, para los abastecimientos de energía, y con una red ferroviaria que ha completado sus ejes radiales con abundantes tramos complementarios y de enlace entre aquellos, Madrid, capital estatal y centro neurálgico del transporte interior modernizado, se configura como un espacio abierto que cumple funciones de cohesión en el plano nacional, y tanto políticamente en el difícil proceso de construcción del Estado liberal -se ha escrito con acierto- cuanto económicamente, al reforzar su papel de nudo estratégico para el abastecimiento y la circulación de productos, para potenciar, en definitiva, los intercambios mercantiles en un mercado interior en formación, que sólo entonces comienza a organizarse unitariamente a escala peninsular.

Durante el despliegue de las siguientes fases de la industrialización - con la electricidad y las industrias químicas y de automoción como estandartes adelantados-, Madrid, desde fechas tempranas del siglo $\mathrm{XX}$, diversificará su tejido productivo, con la multiplicación de iniciativas fabriles y comerciales, al tiempo que contempla el nacimiento de las entidades financieras que, avanzado el novecientos, le otorgarán la condición indiscutida de capital financiera de España. La economía de Madrid reforzará así ahora su poder polarizador en el mapa nacional, principalmente sobre un amplio conjunto de provincias de toda la España 
interior, en un movimiento que acaba discurriendo, a partir de la segunda mitad de la centuria, en doble sentido: la capital y su contorno absorben y atraen recursos e inmigrantes, pero también animan e impulsan el desarrollo de lugares y poblaciones en círculos de radio creciente, en una progresiva ampliación de los límites de influencia de la aglomeración urbana madrileña.

Espacio abierto y centro polarizador: las funciones que con ello ha desempeñado y cumple la economía de Madrid en el conjunto nacional se ampliarán al compás del proceso de internacionalización de la economía española y de su integración en Europa. Las ventajas geográficas y administrativas que de antiguo han cimentado el desarrollo de la economía de Madrid, se revelarán, en el nuevo y ampliado escenario, como nuevas oportunidades logísticas y empresariales. Y Madrid puede cumplir una auténtica función vertebradora del territorio español, en lo económico como en lo geográfico, en lo institucional como en lo cultural.

Es el desenlace - debe repetirse - de un proceso que en torno a 1900 cobra un impulso decisivo. Desde una doble perspectiva es ésta una afirmación comprobable. Entonces adquirirán perfiles mejor dibujados los principales rasgos que desde épocas anteriores han ido decantando la especialización funcional y productiva de la ciudad; y en los decenios interseculares también toda una amplia serie de iniciativas financieras y realizaciones empresariales marcarán con fuerza las líneas de la evolución de la economía de Madrid a lo largo de todo el siglo XX. No resulta hoy difícil resumir lo fundamental en una y otra vertiente, aprovechando lo expuesto ya en otras ocasiones*.

\section{Factores explicativos}

Tres son los factores que, combinadamente, explican la importancia que alcanza la economía de Madrid en el mapa urbano de la España contemporánea; y los tres avanzan en su efectiva influencia alrededor del tiempo aquí acotado.

El primero de dichos elementos no es otro que la situación geográfica que Madrid ocupa en el centro geométrico del territorio peninsular, constituyendo un nudo natural que interconexiona gran parte del resto del territorio. El secular centrifuguismo en la localización de la población española (y portuguesa) desde el siglo XVIII no hará sino contribuir, paradójicamente, a realzar las posibilidades de Madrid, en tanto que centro de una estructura estrellada, cuyas puntas más pobladas se 
fijarán desde entonces en Barcelona, Valencia, Sevilla-Cádiz, Lisboa, Vigo-La Coruña y Bilbao: los seis vértices de un hexágono cuyo núcleo central es polo de atracción, a su vez, de la población de sus anchísimos contornos inmediatos, formados por una docena de provincias. Como es obvio, cuando las líneas y los medios de transporte interior comparativamente mejores - por seguridad, rapidez y capacidad el ferrocarril supone en su momento cambios de proporciones inéditas, no se olvide- se superpongan a los radios que esa figura dibuja, las ventajas que la situación crea a favor de Madrid para articular todo el mercado nacional se harán patentes, y con especial notoriedad a medida que el proteccionismo conceda prioridad a los intercambios comerciales en el propio ámbito nacional. No otra cosa ocurre, ciertamente, en la España de 1900, cuando la red ferroviaria se encuentra, no sólo muy ampliada respecto de la de un tercio de siglo atrás, sino también -repítase- mejor interconexionada merced al reimpulso constructor de los decenios de 1880 y 1890 , y cuando la reciente «demanda social» de protección consiga ganar posiciones duraderas.

La capitalidad politico-administrativa constituye el segundo factor explicativo de lo que ha alcanzado a ser la economía de Madrid. Es cierto que durante mucho tiempo - acaso hasta la España de la Restauración- ese atributo de Madrid no se traducirá en realizaciones económicas de alcance, permaneciendo muy igual a sí misma la ciudad que es más corte que capital de la monarquía hispana, burocrática, rentista, consumidora, sin capacidad de generar impulsos modernizados de cierta fuerza tanto dentro de sus límites cercados como en las comarcas y regiones circundantes. Pero no pueden infravalorarse las posibilidades que ello crea desde el principio. La capitalidad económica, por supuesto, no puede resolverse por decreto, ni tiene por qué acompañar a la principal sede cortesana; pero el emplazamiento de ésta en el centro del territorio peninsular no sólo contribuye a realzar las posibilidades de la situación de la villa que la acoge; por sí misma se revelará, y de modo creciente con el paso del tiempo, como un potencial de oportunidades. Con otras palabras: para la economía de Madrid es determinante su elección como capital de la burocracia pública con competencias en todo el territorio nacional. Así, la capitalidad estatal, ofreciendo servicios de la Administración y generando otros requerimientos muy diversos, desde los educativos a los comerciales, desde los personales a los colectivos, desde los financieros a los de hostelería, desde los inmobiliarios a los de transporte, es la razón del predominio que en la estructura productiva de Madrid ostentará siempre el sector terciario; pero también la capitalidad es factor de atracción de muchas 
instalaciones fabriles, desde la vieja fábrica de moneda y sello, hasta las empresas suministradoras de material y equipos eléctricos. Por lo demás, esa condición de Madrid marcará igualmente su propia fisonomía urbana: todo un territorio metropolitano marcado por la capitalidad, un rasgo que se acentuará precisamente al despuntar el siglo XX, al compás del reforzamiento de la tendencia hacia la centralización estatal.

Los dos mencionados resortes del destino de Madrid acabarán por decidir el tercero, ya sugerido: el sistema radial de transporte y comunicaciones interiores, al que responden básicamente los trazados postal, ferroviario y de carreteras (y del tráfico aéreo regular, más tarde). Sistema que, en todo caso, durante los decenios interseculares va traduciendo en hechos las oportunidades que desde antaño brindaban la situación y la capitalidad, esto es, tanto desde la perspectiva de la integración del mercado nacional, como desde la de la efectiva unificación administrativa del Estado.

Más aun: situación, capitalidad político-administrativa y estructura radial de transportes y comunicaciones generan a través de sus múltiples interacciones otras posiciones ventajosas para la economía de Madrid. Eso es lo que permite entender que la capital concentre la mayor parte de las entidades bancarias con proyección suprarregional, así como el domicilio de un elevado número de grandes sociedades mercantiles, muchas de las cuales no realizan su actividad productiva en la región madrileña. Dicho de otro modo: que la plaza madrileña se convierta desde que el siglo XX inicia su camino en la capital financiera de España y que, simultáneamente, desde los comienzos de esta centuria pueda considerarse a Madrid con propiedad como capital o primer centro decisorio empresarial, no es sino una consecuencia de la suma de oportunidades que ofrecen los tres factores básicos aludidos. Doble capitalidad añadida que intensificará, a su vez, la atracción de nuevas iniciativas e inversiones productivas, hasta desencadenar un proceso acumulativo y autoalimentado de polarización de actividades económicas. La suerte estará entonces definitivamente echada: Madrid, capital económica de España, en sentido pleno y en términos estrictos.

Ininterrumpido proceso, por lo demás, que acaba por conformar un complejo entramado económico cuyos aspectos más característicos pueden agruparse a su vez en dos puntos: por un lado, la preeminencia de Madrid como gran núcleo productor de servicios; por otro, la importancia que también adquiere el sector industrial.

Un primer rasgo distintivo es, en efecto, el predominio del sector terciario. Supremacía del sector terciario que comenzó basándose en los servicios burocráticos, domésticos y comerciales que requería la 
corte y la demanda de consumo, para afianzarse después sobre modernizados servicios a la producción, administrativos y sociales. Esto caracteriza diferencialmente la pauta del crecimiento madrileño - tanto en lo que se refiere a la composición del producto obtenido como en lo que atañe a la distribución sectorial del empleo- frente a las seguidas por otros núcleos de actividad que han protagonizado la industrialización española; acercándola, en cambio, al patrón de estructura sectorial que es compartido por una buena parte de las capitales occidentales europeas (Romas, Bruselas, Amsterdam, París, Londres). Sin que, por lo demás, eso exprese análogos procesos históricos de desarrollo ni iguales funciones económicas a lo largo del tiempo.

La consolidada importancia del sector industrial es un segundo rasgo identificador de la economía de Madrid, especialmente desde que comienza el siglo XX. En efecto, desde el inicio mismo de esta centuria, sin las restricciones infraestructurales de antaño — suelo, agua, transportes-, con nuevas posibilidades de suministros energéticos - comenzando por la hidroelectricidad-, y ampliada también la disponibilidad de recursos financieros, la industria de Madrid se expande, ganando poco a poco posiciones en el conjunto nacional. Así, ya desde los primeros quinquenios del novecientos ofrece un buen ejemplo de esa España que no llega tarde a la cita de la segunda revolución tecnológica, caracterizada por la utilización de la energía eléctrica, la difusión del motor de combustión interna y la modernización de la industria química. Es entonces también cuando la estructura interna del sector comienza a ajustarse a los moldes que han llegado hasta hoy: relativa diversificación, con los únicos grandes vacíos de la industria básica y de la textil, destacada presencia de una amplia gama de sectores manufactureros de segunda transformación y ligados a la demanda final, que es en gran medida la del propio mercado madrileño, y presencia también notoria de actividades intensivas en capital y tecnología avanzada; además, claro está, de una nutrida muestra de industrias de la construcción, con algunas de las mayores empresas de ámbito nacional y con una pléyade de pequeñas empresas poco cualificadas.

\section{Una realidad cambiante}

La economía de Madrid alrededor de 1900 conoce, como ya se ha anticipado, una amplia serie de novedades que contribuirán poderosamente a definir las líneas principales del desarrollo productivo de 
la capital a lo largo de todo el siglo XX. Es entonces, conviene dejarlo de nuevo anotado, cuando comienza a dejarse atrás un Madrid aún predominantemente cortesano y rezagado en las transformaciones que trae consigo durante el ochocientos la producción fabril y los nuevos horizontes en las relaciones mercantiles, dándose paso con el inicio del novecientos a muy apreciables cambios que conformarán pronto las bases socioeconómicas de Madrid como capital económica de España.

Por lo pronto, se trata de una economía que termina el siglo XIX habiendo salvado inveterados obstáculos para su expansión. Efectivamente, al tiempo que con el ferrocarril se elimina el estrangulamiento del tráfico interior de mercancías (objetivo que con la segunda fase intensiva de la construcción de la red, ya se ha dicho, alcanza mayor virtualidad, al realizarse enlaces secundarios que facilitan el acceso y la utilización de aquélla), también se moderniza el transporte marítimo y la comunicación telegráfica, y comienzan a realizarse las primeras instalaciones de teléfonos. Simultáneamente a todo ello, además, se dan pasos decisivos para superar otras limitaciones básicas que constreñían o atenazaban la expansión de la ciudad y de su economía: a saber, la falta de abundante agua para usos domésticos y fabriles, lo reducido del propio casco urbano y la carencia de energía con posibilidades tecnológicas y económicas competitivas. Desde 1858 el abastecimiento de agua ha quedado asegurado, una vez construido el canal de Isabel II. Desde 1868, año en que se derriba la cerca construida con propósitos fiscales casi doscientos cincuenta años antes, la disponibidad del suelo urbano adquiere proporciones inéditas. $Y$ los últimos quinquenios del siglo XIX comenzarán a anticipar la nueva era de la electricidad y el amplísimo horizonte de innovaciones productivas que la misma permitirá.

Nuevas dotaciones de trabajo y capital en la economía de Madrid coincidirán asimismo en los últimos tramos del ochocientos. El crecimiento de la población es muy marcado a lo largo de casi toda la segunda mitad de ese siglo, gracias a una creciente capacidad receptora de emigrantes (durante todo ese tiempo en torno al $45 \%$ de la población censada en Madrid es originaria de otra provincia, proporción que dobla ampliamente las correspondientes a Barcelona y Vizcaya); dinamismo inmigratorio, podría decirse, que sin duda guarda relación con todas las novedades en el desarrollo de la ciudad y también, claro está, con las fuerzas de expulsión que actúan sobre la población de muchas zonas rurales, en particular sobre las familias campesinas más negativamente afectadas por la desamortización de bienes comu- 
nales, desde los años cincuenta, y por la crisis agropecuaria de los ochenta y primeros noventa, después. Junto a ello, durante los años finiseculares se intensificarán también los flujos de capital con el trasvase de capitales formados en América y con nuevas y ampliadas inversiones procedentes de varios países europeos.

Para la economía de Madrid, la suma de todo ello no puede ser sino beneficiosa. Antes de que finalice el siglo, en efecto, los avances son sensibles no sólo en la industria de la construcción y de materiales y equipamientos con ella relacionados -durante décadas enteras nervio de la economía de Madrid-, sino también en varias ramas industriales de importancia creciente en el proceso de modernización económica: la metalurgia, la de artes gráficas y prensa e incluso una naciente industria química, propiamente dicha.

Claro que sigue siendo muy alto el peso de las industrias alimentarias, la mayor parte de ellas aún de reducida dimensión y equipamientos tradicionales; y que se hará cada vez más patente el raquitismo de la industria textil en la estructura manufacturera y fabril de la economía del Madrid de la época. Con todo, las condiciones son paulatinamente propicias para la apertura de nuevas empresas y líneas de actividad. Entre 1879 y 1897 el número total de establecimientos industriales pasará de 962 a 1.221, con aumentos significativamente distintos en el Centro (un 10\%), en el Ensanche (un 20\%) y en el Extrarradio (un 300\%). Y es bien conocido el brillo madrileño en la estadística que recoge la creación de sociedades mercantiles en los años interseculares, ocupando Madrid siempre el primer o segundo puesto en las series que registran por provincias la cuantía de los capitales asociados y el número de sociedades anónimas. Entre estas últimas, la constitución del Banco Hispano americano y del Español de Crédito, en los albores mismos del novecientos, cuando también comienza a ser perceptible cierta predilección del Banco de España por las actividades industriales y comerciales, señalará la importancia de las finanzas madrileñas, cuya hegemonía en ese sentido, aunque apuntada ya bastantes decenios atrás, será a partir de entonces incontestada.

El panorama al concluir el siglo es, en suma, no poco prometedor. La tensión entre lo viejo y lo nuevo es quizá entonces especialmente intensa: "luz fuerte al lado de sombra obscura", verá Baroja en su trilogía sobre el Madrid de esos años, La lucha por la vida. Pero, en todo caso, la situación es ya muy distinta a la de años atrás. Si bien mediado el siglo XIX todavía las transformaciones fundamentales ligadas a la Revolución industrial estaban por llegar a la economía de 


\section{Madrid, Capital económica}

Madrid, hacia 1900 la urbe ha conseguido superar limitaciones estratégicas para el surgimiento de actividades productivas de corte fabril y del terciario moderno; y es una ciudad que, consolidada como capital financiera de país y centro ahora también de la red modernizada de transportes y comunicaciones, refuerza su atracción para multiplicadas sociedades mercantiles. En una palabra, en la coyuntura finisecular la economía de Madrid está presta para concurrir provechosamente a la cita de las novedades tecnológicas y las sendas de crecimiento que se impondrán con el nuevo siglo.

De ahí que pocas fases de la evolución económica y social del Madrid contemporáneo presenten tanto interés para el estudio como las que se suceden durante el primer tercio del siglo. El entramado económico de la ciudad crece, se espesa y diversifica. Se multiplican las instalaciones industriales: ya en 1905, un recuento oficial suma una potencia instalada en la industria madrileña diez veces superior a la que se calculaba para 1885 (26.078 caballos de vapor frente a 2.500). Se desarrollan las ramas en que existía una tradición apreciable, por ejemplo, artes gráficas, metalurgia y alimentación; y despuntan con fuerza sectores representativos de los nuevos tiempos: química y, sobre todo, industria eléctrica, en cuyo dominio se asiste a partir de los años diez al desplazamiento de los motores térmicos por la hidroelectricidad. Aparecen también con cierto dinamismo las primeras empresas del sector de la automoción, tanto de automóviles, ómnibus y camiones, como de aviones y motores de aviación. Por su parte, la construcción y las industrias derivadas conocen un fuerte ritmo de crecimiento, con un auténtico boom durante el decenio de 1920. Década en que se define ya con nitidez un espacio fabril en el Sur, en torno a Arganzuela y a lo largo del ferrocarril de circunvalación, que con la apertura de la estación intermedia de Peñuelas en 1914 (la Imperial es de 1881, cuando se enlaza también la de Delicias) verá multiplicarse en su corto tendido casi dos docenas de apartaderos industriales y de apeaderos de expedición y descarga de mercancías al pie de multiplicadas empresas.

El sector terciario también se expande y renueva. El auge de los servicios financieros es no poco espectacular; situándose Madrid desde los años de la primera guerra mundial en posiciones hegemónicas en el marco nacional, hecho que se acentuará asimismo durante el decenio de 1920, cuando los grandes Bancos establecidos en la capital procedan resueltamente a la apertura ininterrumpida de sucursales por todo el país, unificando con ello de modo efectivo el mercado financiero nacional, con drenaje del ahorro de las más diversas regiones, provincias 
y localidades, y la colocación de esos recursos en los centros y unidades de producción con más elevadas exigencias de financiación ajena. En esa misma década el desarrollo y modernización de la red y de los equipos de telefonía marca un hito en la evolución de la economía de la ciudad, como también lo hacen las mejoras y novedades en el transporte urbano, con la generalización de los tranvías eléctricos y la inauguración del ferrocarril metropolitano, el Metro, en 1919. Incluso en un ámbito tan resistente al cambio como el del comercio minorista, los cambios comienzan a ser apreciables, con la introducción de empresas de carácter capitalista en el comercio madrileño y la aparición de algunos grandes almacenes, aunque desde luego siga dominando la atomización.

Todavía otro hecho sobresaliente puede citarse en este otro corto muestreo: es también en el curso del primer tercio del novecientos, con especial intensidad en los años veinte, cuando Madrid se incorpora al nuevo modelo demográfico, con reducción de las tasas generales $\mathrm{y}$, en particular, las de mortalidad total y mortalidad infantil, registrando entonces la población un crecimiento vegetativo moderado -frente a las tasas negativas de años atrás-, que se añade a las cuantiosas ganancias de habitantes que la acrecida inmigración proporciona (el holgado medio millón de habitantes de 1900 se ha multiplicado por dos en vísperas de la guerra civil). Población numéricamente duplicada cuya residencia urbana - con una cada vez más marcada zonificación social de la ciudad: barrios burgueses, zonas y viviendas obreras- mejora también sus equipamientos básicos, dejando atrás aquel deplorable estado sanitario que todavía en 1902 pudo denunciar Ph. Hauser tras un minuciosos análisis.

\section{Sine ira...}

$\mathrm{Ni}$ «ímpetu imperial» - la expresión es de Santos Juliá- ni voluntaristas políticas discriminatoriamente ventajosas antes o durante el franquismo: Madrid asienta y fortalece su condición de capital económica de España a lo largo del siglo XX como consecuencia del despliegue de oportunidades que durante la España moderna y el siglo XIX han creado un conjunto de condiciones y factores singulares. Oportunidades que -actuando tal vez el ferrocarril como desencadenante último- comenzarán a materializarse con especial notoriedad al despuntar la centuria del novecientos. Es, desde luego, más un «movimiento natural» —otra vez Santos Juliá- que un empeño forzado o artificioso. Algo que también puede decirse, por cierto, de lo cultural —desde la 
creación artística a la investigación científica, desde la presencia de intelectuales al desarrollo de centros tecnológicos-, esa otra dimensión de la capitalidad de Madrid que con el novecientos cobra especial fuerza. No cabe sino concluir así, en efecto, al contemplar con ánimo sólo de estudio la exitosa trayectoria de Madrid a lo largo del novecientos, y no sólo con el encuadre que proporciona la España del siglo XX, sino también situándola en el contexto del crecimiento y la modernización de buena parte de la Europa occidental contemporánea.

Tal vez también por eso - permítase este renglón final- Madrid ha sido durante los pasajes más cruciales de la centuria ahora concluida el corazón de España, como proclamó el verso de Alberti dedicado a la capital resistente en la Guerra Civil. Un corazón solidario.

\section{Notas}

* Dos son los trabajos, separados, por un decenio, que está en la base de lo que aquí se expone. Uno es el incluido en la obra de Jordi Nadal y Albert Carreras (dirs.), Pautas regionales de la industrialización española, Ariel, Barcelona, 1990, páginas 219 a 256. El otro es el que abarca los dos primeros capítulos de José Luis García Delgado (dir.), Estructura económica de Madrid, Cívitas, Madrid, 1999, páginas 3 a 48. A ambos se remite para cotejar las referencias bibliográficas que dan sustento a estas reflexiones. 